\title{
FORUM
}

\section{Auf dem Weg zur variablen Geometrie - Implikationen der EU-Erweiterung für die ESVP und NATO}

\author{
Ralf Roloff*
}

\begin{abstract}
Zu Beginn der deutschen EU-Ratspräsidentschaft stehen grundlegende Fragen der Erweiterung und Vertiefung ganz oben auf der politischen Agenda. Viele Fragen über den weiteren Weg der europäischen Integration sind derzeit noch ungelöst. Zuletzt diskutierten die Staats- und Regierungschefs auf dem Dezembergipfel über zukünftige Erweiterungen der Europäischen Union. Aufnahmefähigkeit, Erweiterungsmüdigkeit und der Ruf nach einer Wiederbelebung des Verfassungsprozesses sowie anderen Möglichkeiten der Vertiefung bestimmen die aktuelle europapolitische Debatte. Der Bericht der Kommission zur Erweiterungsstrategie der EU greift den Aspekt der Aufnahmefähigkeit explizit auf. Welche Bedeutung hat diese Debatte für sicherheitspolitische Entwicklungen in Europa? Schon seit der Irak-Krise 2003 werden die Auswirkungen der Osterweiterung auf die europäische Sicherheitspolitik eingehend diskutiert. Eine generellere Fragestellung lässt sich daraus ableiten: Welche Implikationen ergeben sich aus der EU-Erweiterung für die Europäische Sicherheits- und Verteidigungspolitik (ESVP) und für die NATO?
\end{abstract}

\section{Fragen zur künftigen europäischen Sicherheitsarchitektur}

Vor dem Hintergrund der Irak-Krise 2003 und der EU-Erweiterung 2004 ist nach den Lehren zu fragen, die daraus gezogen wurden, und zwar vor allem im Hinblick auf die bekannten Schwächen der GASP und der ESVP: die mangelhafte Kohärenz, Flexibilität und politische Führung, Zeichen der Desintegration, der politischen und inhaltlichen Divergenzen oder gar das Fehlen von gemeinsamen Standpunkten, um nicht die Spaltungsformel vom ,alten' und ,neuen' Europa zu zitieren. Auf der anderen Seite sind die Beziehungen zwischen der EU und der NATO im Kontext der klassischen ,3 D-Formel' (no discrimination, no duplication, no decoupling) zu diskutieren. Beide Themenkomplexe sind wiederum eng mit der noch offenen Frage verknüpft, wie Erweiterung und Vertiefung in Einklang gebracht werden können. ,Variable Geometrie' oder auch differenzierte Integration sind Schlüsselbegriffe in dieser Debatte. Eine Europäische Union der 27 Mitglieder zwingt uns, auch über flexible Integrationskonzepte jenseits der Vollmitgliedschaft nachzudenken.

Die aktuelle Debatte über die ,Aufnahmefähigkeit ${ }^{{ }^{1}}$ der EU, oder, wie Gideon Rachman es pointiert formuliert ,the death of enlargement “2, wirft jedoch weitere Fragen auf. Welche

* PD Dr. Ralf Roloff, George C. Marshall European Center for Security Studies, Garmisch-Partenkirchen. Dieser Aufsatz gibt ausschließlich die Meinung des Autors wieder und entspricht nicht den offiziellen Standpunkten des George C. Marshall European Center for Security Studies, der U.S. Administration oder der deutschen Bundesregierung.

Der Beitrag basiert auf einem Vortrag anlässlich des vom Institut français des relations internationales und dem Institut für Europäische Politik gemeinsam veranstalteten transatlantischen Workshops ,EU enlargement and the future of transatlantic relations" am 11. Dezember 2006 in Paris.

1 Siehe dazu u.a. Europäische Kommission: Erweiterungsstrategie und wichtigste Herausforderungen für den Zeitraum 2006-2007 mit Sonderbericht über die Fähigkeit der EU zur Integration neuer Mitglieder, 8.11.2006, KOM (2006) 649 endgültig; Europäisches Parlament: Entschließung zu den institutionellen Aspekten der Fähigkeit der Europäischen Union zur Aufnahme neuer Mitgliedstaaten, Rapporteur: Alexander Stubb (2006/2226 (INI) A6-0393/206).

2 Gideon Rachman: The Death of Enlargement, in: The Washington Quarterly 3/2006, S. 51-56. 
Auswirkungen hat ein Erweiterungsstopp der EU für die ESVP und für die NATO? Dies sind zentrale Zukunftsfragen, denn sie ziehen tiefgreifende Konsequenzen für Stabilität und Sicherheit im größeren Europa nach sich. Kann der Stabilisierungs- und Transformationsprozess ohne klar definierte EU-Beitrittsperspektiven vorangebracht werden? Sind die Nachbarländer der EU ohne Aussicht auf eine EU-Mitgliedschaft potenzielle Kandidaten für einen NATO-Beitritt? Zerbrechen diese Staaten oder orientieren sie sich wieder nach Russland - ein Szenario, das insbesondere im Hinblick auf die Ukraine kritisch zu beobachten sein wird. Welche Implikationen ergeben sich für die transatlantischen Beziehungen, für die NATO und die ESVP? Kurzum: Welche Bedeutung haben diese Entwicklungen für die europäische Sicherheitsarchitektur?

Eine mögliche Antwort auf diesen Fragenkomplex führt zurück zum Konzept der ,variablen Geometrie“. Der proklamierte „,death of EU enlargement“ wird gleichzeitig der Beginn der differenzierten Integration in Europa sein. Variable Geometrie wird einerseits als Kooperationsmodell der ESVP jenseits der Vollmitgliedschaft und andererseits als Leitprinzip einer neuen transatlantischen Aufgabenteilung innerhalb der NATO dienen. Der Ansatz der ,variablen Geometrie‘ soll im Folgenden anhand von drei Thesen diskutiert werden.

- These zur internen Entwicklung: Aufgrund der Erweiterung ist die EU gezwungen, die ESVP als Vorreitermodell der variablen Geometrie zu nutzen.

- These zur externen Entwicklung: Der proklamierte „,death of enlargement“ ist der Durchbruch der variablen Geometrie jenseits der EU Mitgliedschaft.

- These zur transatlantischen Entwicklung: Variable Geometrie ist der Beginn einer neuen transatlantischen Arbeitsteilung.

\section{Die ESVP als Vorreitermodell der variablen Geometrie}

Die Sicherheits- und Verteidigungspolitik der EU basierte von Beginn an auf flexiblen Integrationsformen innerhalb und außerhalb der Gemeinschaftsverträge. So wurde das Eurokorps von einer Gruppe von Staaten gegründet, die europäische militärische Fähigkeiten aufbauen wollten. Für EU-Mitglieder, die nicht der NATO angehören, mussten Ausnahmeregelungen definiert werden. Opt-outs und Opt-ins sind innerhalb der ESVP an der Tagesordnung. Wenn man nach einem funktionierenden System der flexiblen Integration oder variablen Geometrie sucht, dann ist die ESVP ein anschauliches Beispiel. ${ }^{3}$ Auch wenn der Vertrag von Nizza eine verstärkte Zusammenarbeit mit ,militärischen oder verteidigungspolitischen Bezügen“ ausschließt, so sieht die Realität anders aus. Schon heute findet eine ,strukturierte Zusammenarbeit" in vielen Bereichen statt, wie sie auch im Verfassungsvertrag vorgesehen ist. Die folgenden Beispiele verdeutlichen die vielschichtigen Ausprägungen der variablen Geometrie:

- Dänemark nimmt nicht an der ESVP sowie der Europäischen Verteidigungsagentur teil (Opt-out Regelung).

- Das Konzept der Battle Groups wird von 20 der 25 Mitgliedstaaten und darüber hinaus von Nicht-Mitgliedern der EU (zum Beispiel Norwegen) umgesetzt.

- Die 13 Battle Groups sind teils unilateral und teils multilateral zusammengesetzt.

- OCCAR (Organisation Conjoint de Cooperation en matiere d'Armement), die zum effizienten Management von gemeinsamen Rüstungsvorhaben gegründet wurde, hat sechs Mitglieder: Belgien, Deutschland, Frankreich, Großbritannien, Italien und Spanien.

3 Vgl. Charles Grant: Europe's blurred boundaries - Rethinking enlargement and neighbourhood policy, London 2006. 
- 22 Staaten haben einen neuen Verhaltenskodex im Bereich der Rüstungsbeschaffung unterzeichnet (Dänemark, Spanien und Ungarn entschieden sich für ein Opt-out).

- Die Unterzeichnerstaaten des letter of intent umfassen ebenfalls nicht die gesamte EU-27. Deutschland, Frankreich, Großbritannien, Italien, Schweden und Spanien bringen 80 Prozent der gesamten Militärausgaben und 98 Prozent der militärischen Forschungs- und Entwicklungsausgaben in der EU auf.

- Das Eurokorps ist immer noch ein rein westeuropäisches Korps (Belgien, Deutschland, Frankreich, Luxemburg und Spanien).

- Das Europäische Sicherheits- und Verteidigungskolleg besteht aus einem Netzwerk nationaler Institute, Akademien sowie dem Institut der Europäischen Union für Sicherheitsstudien. Es umfasst derzeit zwölf Mitgliedstaaten (Belgien, Deutschland, Finnland, Frankreich, Großbritannien, Italien, Litauen, Niederlande, Österreich, Schweden, Spanien und Ungarn).

Angesichts der unterschiedlichen militärischen Fähigkeiten und Ambitionen sowie der divergierenden regionalen Sicherheitsinteressen der 27 Mitgliedstaaten sind flexible Integrationsformen entstanden, die sich auch künftig neu formieren können. Heute gibt es daher in Europa vor allem multinational zusammengesetzte Gefechtsverbände mit integrierten Planungsstäben, aber nationalen Sub-Kontingenten, wie zum Beispiel Eurofor, Euromarfor und die Multinational Peace Force Southeastern Europe. Nicht zuletzt auch aus finanziellen Gründen gibt es zudem beim Aufbau der militärischen Fähigkeiten bereits eine Reihe von Kooperationsprojekten:

- Das Airbus A400M-Projekt wird von sechs EU-Mitgliedstaaten und der Türkei getragen.

- Italien und Deutschland arbeiten zusammen mit den USA an der Entwicklung des Mittelstrecken-Raketenabwehrsystems MEADS (Medium Extended Air Defence System).

- Frankreich und Großbritannien führen Gespräche über ein gemeinsames FlugzeugträgerProjekt.

- Einige Mitgliedstaaten könnten sich zudem zu einer Zusammenlegung von Unterstützungsoperationen für bestimmte Teile der Ausrüstung und logistische Organisation entschließen. Dies bietet sich zum Beispiel für Länder an, die Transportflugzeuge von russischen und ukrainischen Unternehmen im Rahmen von SALIS (strategic airlift interim solution) mieten.

Offenkundig ist also die ESVP ein Beispiel für variable Geometrie. Weniger klar gesehen wird jedoch, dass die Erweiterung der flexiblen Integration innerhalb der ESVP neuen Auftrieb verliehen hat. Schließlich sind ,Flexibilisierungsklauseln', wie sie im Vertrag von Nizza und weitreichender im Verfassungsvertrag zu finden sind, Maßnahmen zur institutionellen Anpassung und Vorbereitung auf die Erweiterung der Europäischen Union gewesen. Das Prinzip der Intergouvernementalität der zweiten Säule erleichtert den Umgang mit diesem hohen Grad an Flexibilität. Dass einzelne Mitgliedstaaten Verantwortung für die Weiterentwicklung der ESVP und der GASP übernehmen können, ist eine der „key prerequisites in enabling an EU of 25 or more member states to avoid policy stalemate and to promote effective action. “4 Die Ständige Strukturierte Zusammenarbeit, wie sie im 23. Protokoll des Verfassungsvertrags festgeschrieben ist, würde die Grundlage für eine dauerhafte Form der variablen Geometrie bilden. Sie könnte als Ausgangspunkt für die Entwicklung gemeinsamer militärischer Strukturen fungieren und damit die derzeitige Form kombinierter Fähigkeiten ergänzen. Ein weiteres Element variabler Geometrie spiegelt sich im Art. I-41 Abs. 5

4 Franco Algieri / Thomas Bauer / Klaus Brummer: Options for the further development of CFSP and ESDP without a European Constitution, Gütersloh 2005, S. 16. 
des Verfassungsvertrags wider, nach dem der Rat eine Gruppe von Mitgliedstaaten beauftragen kann, ,zur Wahrung der Werte der Union und im Dienste ihrer Interessen“ Missionen durchzuführen. Dieser Artikel setzt auf ,Koalitionen der Willigen und Fähigen“. Letztlich zielen all diese Flexibilisierungsmaßnahmen darauf ab, die ESVP einer EU-27 handlungsfähig und effektiv zu gestalten.

\section{Variable Geometrie jenseits der EU-Mitgliedschaft}

Einen ,Ring von Freunden“ in der europäischen Nachbarschaft zu schaffen ist ein strategisches Ziel der Europäischen Union. Diese Zielsetzung schlägt sich in der Europäischen Sicherheitsstrategie sowie in der Europäischen Nachbarschaftspolitik (ENP) nieder, die als Ersatz für die Erweiterungspolitik dienen soll. Grundlegende Idee der ENP ist es, eine Annäherung zwischen den Nachbarstaaten und der EU zu ermöglichen. Die ENP basiert auf jeweils individuell ausgearbeiteten Aktionsplänen und auf einem Konzept der variablen Geometrie oder auch der flexiblen Integration. Sie bietet den Nachbarländern, je nach Themenfeld und Kapazitäten der in Frage kommenden Länder, eine Teilhabe an bestimmten EU-Politiken und Programmen an, wobei die Teilnahme am Binnenmarkt als stärkster Anreiz gesehen wird. Der Ansatz der individuellen Aktionspläne ist insbesondere mit dem Konzept der ,positiven Konditionalität‘ verknüpft. Grundsätzlich kann ein ,Ring von Freunden` auf diese Weise geschaffen werden, vorausgesetzt die Nachbarländer sind von der Substanz der ENP nicht allzu enttäuscht. Um dem vorzubeugen, sollten die Anreize der ENP verbessert werden. Ein mögliches zusätzliches Angebot könnte die Partizipation an Initiativen der ESVP sein - nicht als Vollmitglieder, aber eben als weitere Akteure im bereits bestehenden System der variablen Geometrie. Diese Idee hat Charles Grant in seinem Konzept der ,Sicherheitspartnerschaften" ausgearbeitet. ${ }^{5}$

Die Beteiligung von ENP-Ländern und Ländern des westlichen Balkans an ESVP- Missionen könnte schon bald Realität werden. ENP-Länder haben bereits Personal in ESVP-Operationen entsendet: Die Ukraine beteiligte sich an EU-Polizeimissionen in Bosnien und Mazedonien, während Marokko Truppen im Rahmen der EUFOR in Bosnien stationiert hat. Die Partnerländer könnten über die Entsendung von Personal und Truppen hinaus auch am Management von Missionen beteiligt werden. ENP-Partner könnten zudem in den Battle Groups mitwirken, wobei die jeweiligen militärischen Fähigkeiten berücksichtigt werden müssten. So könnten ENP-Länder vor allem in die multinationalen Battle Groups eingegliedert werden. Auch dafür gibt es bereits konkrete Beispiele. Die Ukraine wirkt am PolnischUkrainischen Peace Force Battalion, die im Rahmen der KFOR aktiv ist, mit. Die Türkei, Albanien und Mazedonien kooperieren mit Bulgarien, Griechenland, Italien und Rumänien im Rahmen der Multinational Peace Force Southeastern Europe.

Erst kürzlich hat die Kommission in einer Mitteilung vorgeschlagen, die Teilnahme von ENP-Ländern an Gemeinschaftsagenturen und -programmen generell zu ermöglichen. ${ }^{6}$ Dazu gehören alle Agenturen der zweiten Säule, wie das Institut der Europäischen Union für Sicherheitsstudien in Paris, das Satellitenzentrum der Europäischen Union und die Europäische Verteidigungsagentur. Die Interaktion von nach Paris entsandten Wissenschaftlern kann zu einem verstärkten strategischen Dialog mit den ENP-Ländern beitragen. Die Teilnahme an Aktivitäten der Europäischen Verteidigungsagentur eröffnet ENP-Ländern die Möglichkeit, eine engere Kooperation mit EU-Partnern in den Bereichen militärische Fähig-

5 Grant: Europe's blurred boundaries, S. 66.

6 Europäische Kommission: Mitteilung über das allgemeine Konzept zur Ermöglichung einer Beteiligung von ENP-Partnerstaaten an Gemeinschaftsagenturen und -programmen, KOM (2006)724 endgültig. 
keiten, Beschaffung und Rüstung einzugehen. Zudem wäre es nützlich, eine strategische Gemeinschaft von Sicherheitsexperten aus EU-Mitgliedsländern, den ENP-Ländern sowie dem Westlichen Balkan zu stärken. Der Ansatz der ESVP-Orientierungskurse des Europäischen Sicherheits- und Verteidigungskollegs (ESDC) ist ein erster Schritt in diese Richtung. Angebote für Teilnehmer aus ENP-Ländern und dem Westlichen Balkan könnten zu einer Stärkung dieser strategischen Gemeinschaft beitragen. Eine weitere sinnvolle Maßnahme zur wissenschaftlichen Vernetzung bestünde in der gelegentlichen Einbindung nationaler Sicherheitsakademien aus den Nachbarländern in die Aktivitäten des ESDC. Durch das Angebot von Sicherheitspartnerschaften und andere Partizipationsmöglichkeiten in ESVP-Aktivitäten könnte das bereits bestehende ESVP-System der variablen Geometrie und der Ständigen Strukturierten Zusammenarbeit in die Nachbarschaft der EU exportiert werden. Deshalb scheint die Annahme durchaus berechtigt, dass ,the death of enlargement“ der Durchbruch für flexible Kooperationsformen jenseits der EU-Mitgliedschaft ist. Zurzeit soll die verstärkte Zusammenarbeit als Ausnahme und „nur als letztes Mittel“ (Art. 43a EUV) eingesetzt werden. In Zukunft wird diese Ausnahme aber wohl eher zur Regel werden!

Aus russischer Perspektive könnte dieser Ansatz der variablen Geometrie, vor allem die Einbindung der Ukraine und Georgiens, als Bedrohung wahrgenommen werden. Aber das ist schließlich schon jetzt der Fall. Denn Russland ist offensichtlich wenig erfreut darüber, dass die Europäische Union ihre außen- und sicherheitspolitischen Aktivitäten in die gemeinsame Nachbarschaft ausweitet. Wenn die EU als aktiver und engagierter Partner in der Region auftritt und ein verstärktes Engagement in Sachen Konfliktmanagement beweist, so wird die Ernsthaftigkeit der ENP und der eingebetteten Sicherheitspartnerschaften deutlich werden.

\section{Variable Geometrie als Motor einer neuen transatlantischen Arbeitsteilung}

Angesichts dieser Entwicklungen im größeren Europa stellt sich die Frage nach den Implikationen für die Kooperation zwischen EU und NATO und für die transatlantischen Beziehungen. Ein flexibler Kooperationsansatz im Rahmen der ESVP bedeutet nicht notwendigerweise Rivalität oder gar das Ende der NATO. Die oben beschriebenen Dimensionen der variablen Geometrie können vielmehr als konstruktive Handlungsanstöße verstanden werden, die eine Neuauflage der ,3D-Debatte ‘ begünstigen.

Keine Diskriminierung: Die Ausdehnung der flexiblen ESVP-Kooperation jenseits der EU-Beteiligung ist natürlich keine Exklusivveranstaltung! Die meisten militärischen Kooperationen sowie Aktivitäten im Bereich Rüstung und Beschaffung beziehen sowohl EU- als auch NATO-Mitglieder ein. Außerdem sind die USA an Projekten und Programmen beteiligt, zum Beispiel im Fall von MEADS.

Keine Duplizierung: Alle Länder verfügen nur über einen einfachen Streitkräftebestand (single set of forces). Militärische Fähigkeiten müssen also im Einklang mit NATO-Standards entwickelt werden und tragen somit auch zu einer Aufwertung der NATO-Kapazitäten bei. Die meisten nationalen Beiträge zur NATO Response Force (NRF) sind gleichzeitig auch Bestandteil der Battle Groups der EU. Nicht zuletzt die knappe Bemessung vieler Militärhaushalte erfordert diese Strategie des ,doppelten Einsatzes ' militärischer Fähigkeiten.

Keine Abkopplung: Das Prinzip der variablen Geometrie ermöglicht ESVP Missionen auch dort durchzuführen, wo die NATO nicht aktiv ist oder wo sich die EU nicht als Ganzes engagiert. Könnte nicht so eine Aufgabenteilung zwischen NATO und EU insbesondere im Bereich der zivilen Krisenbewältigung entstehen?

Die Ausdehnung flexibler Integration könnte die NATO in einen Werkzeugkasten für ESVP- und US-Missionen umfunktionieren. Aus der von Karl W. Deutsch entwickelten 
Idee der ,Sicherheitsgemeinschaft" ${ }^{\text {“7 }}$ würde eine flexible Sicherheitsinstitution, die eher auf gemeinsamen Interessen basiert als auf gemeinsamen Werten. Im Rahmen dieses, Werkzeugkasten-Ansatzes ' würde die NATO zu einem flexiblen Instrument; wobei die jeweiligen Einsätze die Zusammensetzung der Koalitionspartner bestimmen. ${ }^{8}$ Informelle Koalitionen, ad-hoc-Gruppierungen und flexible Ansätze sind die charakterisierenden Stichworte. Hieraus ergibt sich ein neues und pragmatischeres transatlantisches Bündnis, in dessen Rahmen die USA eher bilaterale, regionale und weniger multilaterale Strategien verfolgen, während die EU gemeinsame Fähigkeiten auch für ihre eigenen Zwecke nutzen kann. So wird in Zukunft aus dem Verteidigungsbündnis ein politisches Forum, in dem die gemeinsamen transatlantischen Interessen zu identifizieren, zu diskutieren und multilateral zu definieren sein werden. Deshalb ist es zwingend notwendig, die EU-NATO-Beziehungen wiederzubeleben - zumindest aber sie pragmatischer zu gestalten. Momentan wirkt ,Berlin plus ' doch eher wie ,Berlin minus‘. Mehr Flexibilität auf beiden Seiten könnte „Nahtoderfahrungen“ der NATO (so der ehemalige NATO-Botschafter der USA, Nicholas Burns), wie wir sie zu Hochzeiten der Irak-Krise 2003 erlebt haben, verhindern. Den passenden Leitspruch einer flexiblen EU-NATO-Kooperation könnte Bill Clintons , assertive multilateralism ' liefern: „On our own where we must, together where we can."

Alles in allem ist abzusehen, dass eine der strategisch wichtigsten Implikationen der Erweiterung ein unausweichlicher Trend zur variablen Geometrie ist. Was lange als, letztes Mittel ' oder zweitbeste Lösung zur Überwindung von Ineffizienz und politischen Blockaden betrachtet wurde, avanciert zum Katalysator für Sicherheit im größeren Europa, das mit zunehmend komplexen Sicherheitsrisiken konfrontiert ist.

Übersetzung aus dem Englischen von Kristina Kurze.

7 Karl W. Deutsch: Political Community and the North Atlantic Area, Princeton 1957.

8 Andrew Michta: Transatlantic troubles, in: National Interest Nr. 86, November/Dezember 2006, S. 62-66. 